\title{
Article
}

\section{Forming double-barred galaxies from dynamically cool inner disks}

Du, Min, Shen, Juntai and Debattista, Victor P

Available at https://clok.uclan.ac.uk/12521/

Du, Min, Shen, Juntai and Debattista, Victor P orcid iconORCID: 0000-00017902-0116 (2015) Forming double-barred galaxies from dynamically cool inner disks. The Astrophysical Journal, 804 (2). p. 139. ISSN 1538-4357

It is advisable to refer to the publisher's version if you intend to cite from the work. http://dx.doi.org/10.1088/0004-637X/804/2/139

For more information about UCLan's research in this area go to

http://www.uclan.ac.uk/researchgroups/ and search for < name of research Group>.

For information about Research generally at UCLan please go to http://www.uclan.ac.uk/research/

All outputs in CLoK are protected by Intellectual Property Rights law, including Copyright law. Copyright, IPR and Moral Rights for the works on this site are retained by the individual authors and/or other copyright owners. Terms and conditions for use of this material are defined in the policies page.

\section{CLoK}

Central Lancashire online Knowledge www.clok.uclan.ac.uk

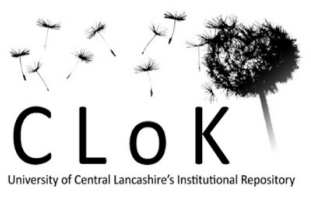




\title{
FORMING DOUBLE-BARRED GALAXIES FROM DYNAMICALLY COOL INNER DISKS
}

\author{
Min Du ${ }^{1,2}$, Juntai Shen ${ }^{1}$, and Victor P. Debattista ${ }^{3}$ \\ ${ }^{1}$ Key Laboratory of Research in Galaxies and Cosmology, Shanghai Astronomical Observatory, Chinese Academy of Sciences, \\ 80 Nandan Road, Shanghai 200030, China; jshen@shao.ac.cn \\ ${ }^{2}$ University of China Academy of Sciences, 19 A Yuquanlu, Beijing 100049, China \\ 3 Jeremiah Horrocks Institute, University of Central Lancashire, Preston, PR1 2HE, UK \\ Received 2014 December 16; accepted 2015 February 23; published 2015 May 12
}

\begin{abstract}
About one-third of early-type barred galaxies host small-scale secondary bars. The formation and evolution of such double-barred (S2B) galaxies remain far from being well understood. In order to understand the formation of such systems, we explore a large parameter space of isolated pure-disk simulations. We show that a dynamically cool inner disk embedded in a hotter outer disk can naturally generate a steady secondary bar while the outer disk forms a large-scale primary bar. The independent bar instabilities of inner and outer disks result in long-lived doublebarred structures whose dynamical properties are comparable to those in observations. This formation scenario indicates that the secondary bar might form from the general bar instability, the same as the primary bar. Under some circumstances, the interaction of the bars and the disk leads to the two bars aligning or single, nuclear, bars only. Simulations that are cool enough of the center to experience clump instabilities may also generate steady S2B galaxies. In this case, the secondary bars are "fast," i.e., the bar length is close to the co-rotation radius. This is the first time that S2B galaxies containing a fast secondary bar are reported. Previous orbit-based studies had suggested that fast secondary bars were not dynamically possible.
\end{abstract}

Key words: galaxies: evolution - galaxies: formation - galaxies: kinematics and dynamics - galaxies: stellar content - galaxies: structure

Supporting material: animations

\section{INTRODUCTION}

Double-barred (S2B) galaxies, consisting of a small-scale secondary bar embedded in its large-scale primary counterpart, were first described nearly $40 \mathrm{yr}$ ago (de Vaucouleurs 1975). Statistics of the fraction of S2Bs among early-type galaxies have been obtained from optical (Erwin \& Sparke 2002; Erwin 2004) and infrared (Laine et al. 2002) observations that showed that about one-third of early-type barred galaxies are S2Bs. We still lack systematic surveys of later Hubble types because of their stronger dust extinction, especially in the central region (Erwin 2005).

Observations (Buta \& Crocker 1993; Friedli \& Martinet 1993; Corsini et al. 2003) have shown that the two bars rotate independently, which was expected from numerical simulations (e.g., Shlosman \& Heller 2002; Debattista \& Shen 2007, hereafter DS07). In general, nested bars cannot rotate through each other rigidly (Louis \& Gerhard 1988). In the potential of two independently rotating bars, the orbits may not be closed in any reference frame. Maciejewski \& Sparke (1997, 2000) and Maciejewski \& Athanassoula (2007) studied the orbits based on the concept of loops, which is a family of orbits whose population of particles return to the same curve, but not to the same position, when the two bars return to the same relative orientation. They also showed non-rigid rotation for loops. Dynamically decoupled secondary bars in S2Bs have been hypothesized to be a mechanism for driving gas past the inner Lindblad resonance (ILR) of the primary bars to feed the supermassive black holes that power active galactic nuclei (AGNs; Shlosman et al. 1989, 1990).

Other works studied S2Bs from a purely kinematical point of view. de Lorenzo-Cáceres et al. (2008) presented the 2D stellar velocity and velocity dispersion maps of a sample of four S2Bs, based on observations with the SAURON integral-field spectrograph. The high quality velocity dispersion maps reveal two local minima, located near the ends of the secondary bar of each galaxy. They suggested that these $\sigma$-hollows appear because of the contrast between the velocity dispersion of a hotter bulge and the secondary bar, as the secondary bar is dominated by ordered motion and thus has a low $\sigma$.

The formation of S2Bs has been studied by numerical simulations. The best-known scenario for forming independently rotating double bars was proposed by Friedli \& Martinet (1993): a pre-existing large-scale bar drives gas inflow into the central kiloparsec of a galaxy; once sufficient gas has accumulated, it becomes bar-unstable and a dynamically decoupled (gaseous) secondary bar forms (see also Combes 1994; Heller et al. 2001; Shlosman \& Heller 2002; Englmaier \& Shlosman 2004). However, the S2B structures forming from gas are short-lived and gas dominated; this cannot explain the observed high abundance of S2Bs in gaspoor early-type galaxies (Petitpas \& Wilson 2004). However, recently 3D $\mathrm{N}$-body+hydrodynamical simulations by Wozniak (2015) formed long-lived S2Bs that might provide a better description of this process. The new stars form from central gas accumulations producing a dynamically cool inner disk. The stellar populations are in qualitative agreement with the observations of de Lorenzo-Cáceres et al. (2013) in that the secondary bars are a few gigayears younger than their primary counterparts.

Rautiainen \& Salo (1999) and Rautiainen et al. (2002) reported the formation of S2Bs in purely collisionless studies, although these secondary bars often have a "vaguely spiral shape." DS07 and Shen \& Debattista (2009) performed wellresolved simulations of long-lived S2Bs with a pre-existing rapidly rotating pseudobulge without any gas. Finally, some simulations have indicated that the dark matter halo may 
sometimes play a role in generating S2Bs (Heller et al. 2007; Saha \& Maciejewski 2013). Thus the conditions of S2B formation are still not well understood. Nevertheless, this variety of simulations shows that gas is not required to form secondary bars.

Here we present new three-dimensional (3D) $N$-body simulations that successfully generate S2Bs from simple and natural initial conditions. We form long-lived S2B structures in pure-disk simulations by choosing different initial parameters for the inner and outer parts of the disk. Starting from different dynamical conditions, the inner and outer disks generate independent bar instabilities that result in a high probability of S2B formation. The paper is organized as follows. In Section 2 we describe the model setup. In Section 3 we summarize the results of exploring the parameter space. In Section 4 we show case studies of S2B galaxies. A comparison with single-barred galaxies is presented in Section 5. Finally, in Section 6 we summarize and discuss the implications of this work.

\section{MODEL SETUP}

The $N$-body simulations are evolved with a 3D cylindrical polar grid code, GALAXY (Sellwood \& Valluri 1997; Sellwood 2014). All models consist of a live exponential disk (scale length $R_{d}$, stellar mass $M_{d}$ ) and a rigid dark matter halo, with no gas present. The simulations use units where $G=M_{0}=R_{d}=V_{0}=T_{0}=1$. The key point is that a dynamically cool inner disk is used to generate strong smallscale bar instabilities. As shown in Equation (1), the initial Toomre- $Q$ profile is roughly constant at 2.0 in the outer disk $(R>1.75)$, while in the inner disk it decreases gradually to $b_{Q}$ following a quadratic curve:

$$
Q(R)= \begin{cases}\left(2.0-b_{Q}\right)\left(\frac{R}{1.75}\right)^{2}+b_{Q} & (R \leqslant 1.75) \\ 2.0 & (R>1.75) .\end{cases}
$$

Thus the outer disk is dynamically hot, while the inner part of the disk is dominated by ordered motion. The only free parameter, $b_{Q}$, can be used to set the dynamical temperature of the inner disk. The initial thickness $z_{0}$ is 0.1 all over the disk.

In this study, the halo potential is logarithmic

$$
\Phi(r)=\frac{1}{2} V_{h}^{2} \ln \left(r^{2}+r_{h}^{2}\right),
$$

with $V_{h}=0.6$ and $r_{h}=15$. Because the models are very disk dominated and the halo density is low near the center where the secondary bar dominates, we expect that the angular momentum transfer between the bar and the halo should be less important than models in Debattista \& Sellwood (2000) and Athanassoula (2003). The rigid halos are useful for studying the complicated co-evolution of the two bars without the additional weaker evolution introduced by a live halo by allowing high mass resolution in the nuclear regions. The initial disk has $4 \times 10^{6}$ equal-mass particles. A possible scaling to physical values is $M_{0}=4.0 \times 10^{10} M_{\odot}$ and $R_{d}=3.0 \mathrm{kpc}$, which gives a velocity unit of $V_{0}=\left(\frac{G M_{0}}{R_{d}}\right)^{1 / 2} \simeq 239 \mathrm{~km} \mathrm{~s}^{-1}$ and a time unit of $T_{0}=R_{d} / V_{0} \simeq 12.3 \mathrm{Myr}$. The force resolution (softening) is 0.01 (corresponding to $30 \mathrm{pc}$ ). The forces in the radial direction are solved by direct convolution with the

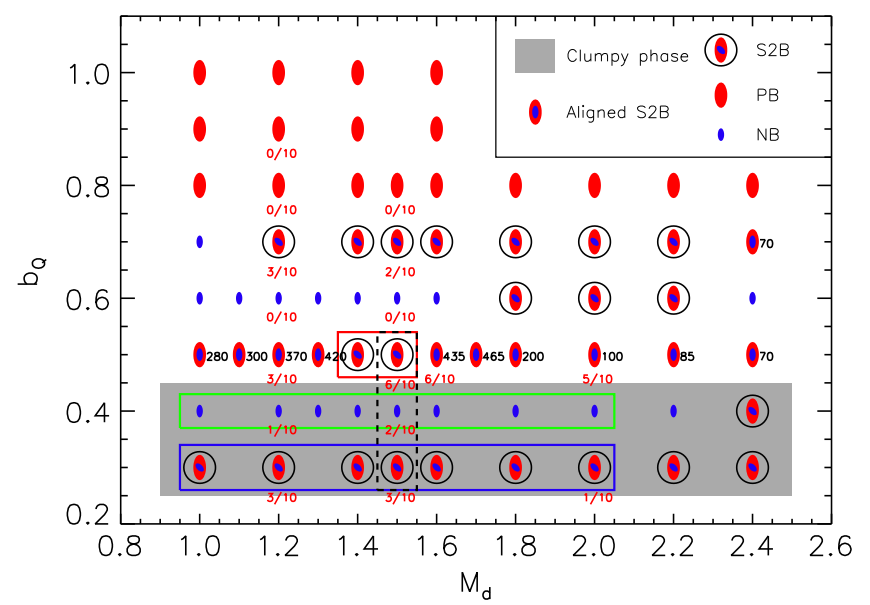

Figure 1. Exploring the parameter space of $b_{Q}$ and $M_{d}$. The long-lived doublebarred, single-barred, and nuclear-barred simulations are marked as "S2B," "PB", and "NB," respectively. The simulations whose two bars align with each other during the simulation are marked as "aligned S2B." The black numbers at the right side of aligned S2Bs are the times when the alignment finishes. The shaded region shows the range of $b_{Q}$ and $M_{d}$ when the inner disk suffers strong clump instabilities at the beginning. The red, green, and blue rectangles highlight the subsamples of standard S2B, clumpy S2B, and NB models, while the dashed black rectangle marks the three models with the same disk mass to compare their evolution of their surface density. The results of the stochasticity test are shown as the fractions in red.

Greens function, while the vertical and azimuthal forces are obtained by fast Fourier transform. We use grids measuring $N_{R} \times N_{\phi} \times N_{z}=58 \times 64 \times 375$ but have verified that increasing resolution does not affect our results by doubling $N_{R}$ and $N_{\phi}$. The vertical spacing of the grid planes was $\delta z=0.01$. Time integration used a leapfrog integrator with a fixed time step $\delta t=0.04$ corresponding to about $0.5 \mathrm{Myr}$.

\section{EXPLORING THE PARAMETER SPACE}

Based on linear bar-formation theory (Toomre 1981), the modes of bar formation are standing waves in a cavity, akin to the familiar modes of organ pipes and guitar strings. To form S2B systems, we strive to build a disk with independent bar instabilities in the inner and outer regions. The responsiveness of the disk can be enhanced by decreasing $Q$, increasing surface density, and reducing disk thickness (Sellwood 1989). Therefore, we explore the parameter space of $b_{Q}, M_{d}$, and thickness. A set of simulations (not presented here) show that reducing the thickness of the inner disk does not strongly affect the formation of S2Bs. The results of simulations with varying $b_{Q}$ and $M_{d}$ are shown in Figure 1. We classify the outcomes into five types based on their formation history and final morphology. There are three types of S2Bs: standard S2Bs, clumpy S2Bs, and aligned (or coupled) S2Bs. Aligned S2Bs are unstable S2Bs whose two bars eventually couple into alignment after a few Gyr. The steady S2Bs forming from a violent clumpy phase when $b_{Q} \lesssim 0.45$ are termed clumpy S2Bs. S2Bs not experiencing a clumpy phase are termed "standard" S2Bs. The two types of single-barred models are divided into large-scale primary single-barred models (PBs) and nuclear-barred models (NBs) depending on the size of the bar. If the semimajor axis is less than 1.0, we classify it as a $\mathrm{NB}$, otherwise, it is a PB.

The chaotic nature of disks leads to significant stochasticity (Miller 1964; Sellwood \& Debattista 2009). Because of the 

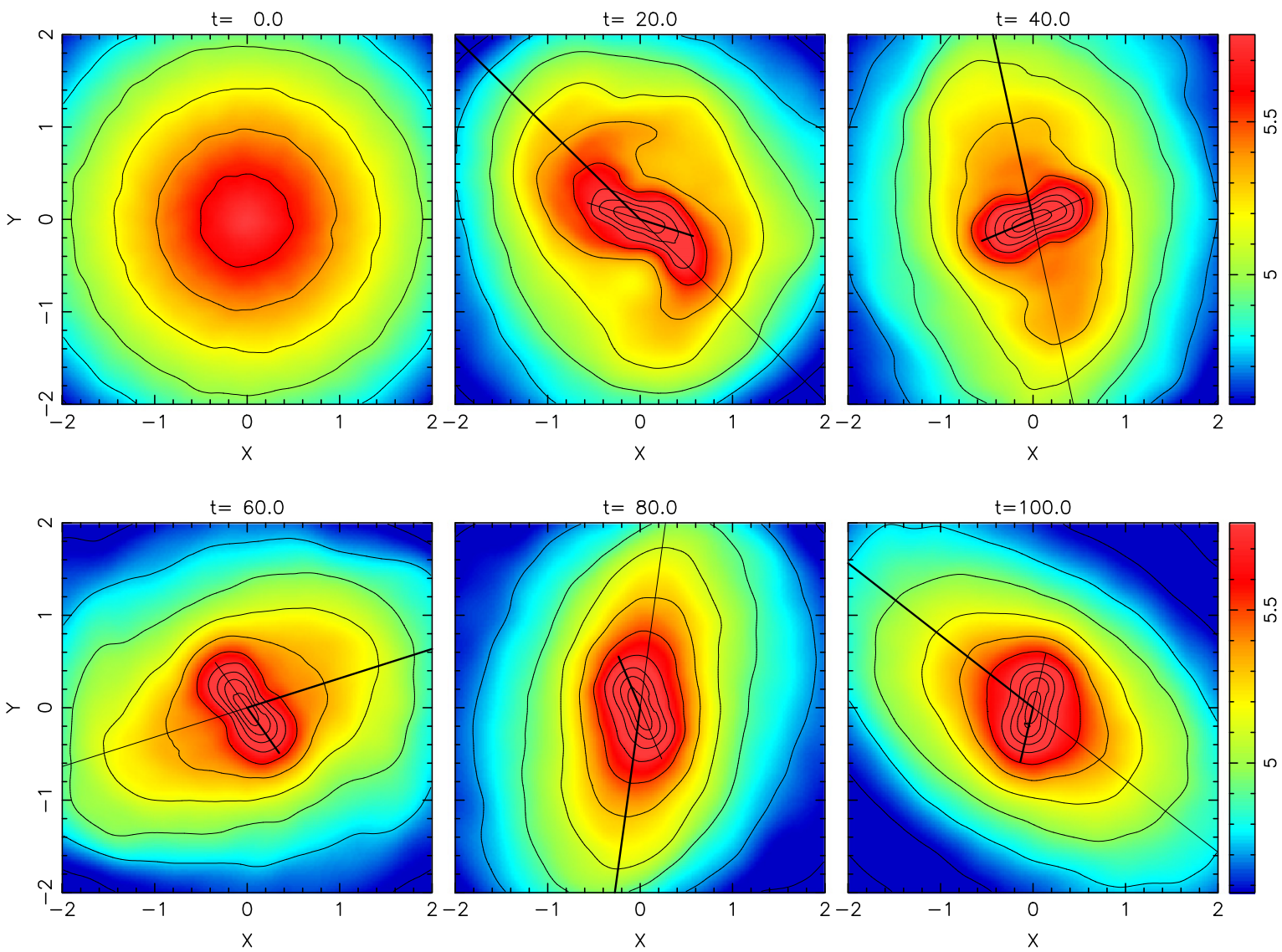

Figure 2. Face-on images of the S2B simulation at various times, with isodensity contours superposed. The contours are equally separated in logarithm, showing the formation process of the S2B structure over the interval $0 \leqslant t \leqslant 100$. The surface density contours have been smoothed with an adaptive kernel (Silverman 1986). The short and long straight lines mark the major axes of the secondary bar and the primary bar, respectively.

(An animation of this figure is available.)

interaction of multiple non-axisymmetric components, the simulations presented here are more stochastic than typical simulations involving one bar. Therefore, we test the degree of stochasticity for a subsample of models by changing only the random seed when generating the particle initial conditions. The results are shown as the fractions in red in Figure 1, whose denominator corresponds to the total number of simulations we have run, while the numerator is the number of simulations forming steady S2Bs, including standard S2Bs and clumpy S2Bs. The fractions give an approximate probability for forming long-lived S2Bs. When S2Bs fail to form, the outcomes can be either aligned S2Bs, or single bars, either NBs or PBs.

\section{THE FORMATION AND EVOLUTION OF S2B GALAXIES}

As shown in Figure 1, the most important condition for S2B formation from independent bar instabilities is given by the $Q$ profile. When $b_{Q} \gtrsim 0.8$, S2Bs do not form for a large range of disk mass. Instead most of such simulations form PBs only.

\subsection{A Standard S2B Galaxy}

The model shown in Figure 2 is a standard S2B. The initial central dynamical temperature parameter, $b_{Q}$, is set to 0.5 . In order to be sufficiently massive for bar instabilities, the mass of the disk is $M_{d}=1.5 M_{0}=6.0 \times 10^{10} M_{\odot}$. Figures 2 and 3 give an overview of the formation of the S2B over 100 time units $(1.23 \mathrm{Gyr})$. The time evolution of the amplitudes of the primary bar $\left(A_{\text {prim }}\right)$ and the secondary bar $\left(A_{\mathrm{sec}}\right)$ is shown in Figure 4.

From both analytical calculations and $\mathrm{N}$-body simulations, we know that the length, strength, and pattern speed reduction of bars are strongly affected by the angular momentum exchange with the outer disk (e.g., Lynden-Bell \& Kalnajs 1972; Athanassoula 2003). Because of the low initial $Q$, the inner disk starts with strong $m=2$ instabilities $(t=0-20$, Figure 3) with a high pattern speed $\Omega_{\mathrm{sec}} \sim 0.80$. A small bar forms extending roughly to its co-rotation radius $R_{\mathrm{CR}} \sim 1.0$, which indicates that it forms via the usual bar instability (Toomre 1981). As time goes on, the small bar sheds angular momentum and traps particles from the disk further out, which happens rapidly as the dynamical time scale is short in the inner disk. In this period $(t=0-50), A_{\mathrm{sec}}$ constantly increases, while the pattern speed of the secondary bar declines to $\Omega_{\mathrm{sec}} \sim 0.50(t=40-60)$.

The primary bar forms a little later and slower than the secondary bar through a quite similar process. It starts with $\Omega_{\text {prim }} \sim 0.37(t=20-40)$ and declines to $\sim 0.18$ at $t=60-80$. From the evolution of $A_{\text {sec }}$ (Figure 4), we can see that the secondary bar is significantly weakened by the formation of the primary bar, so that it can no longer extend to its co-rotation radius. It takes more than 300 time units for $A_{\text {sec }}$ to settle to a steady state, while the change of the primary 

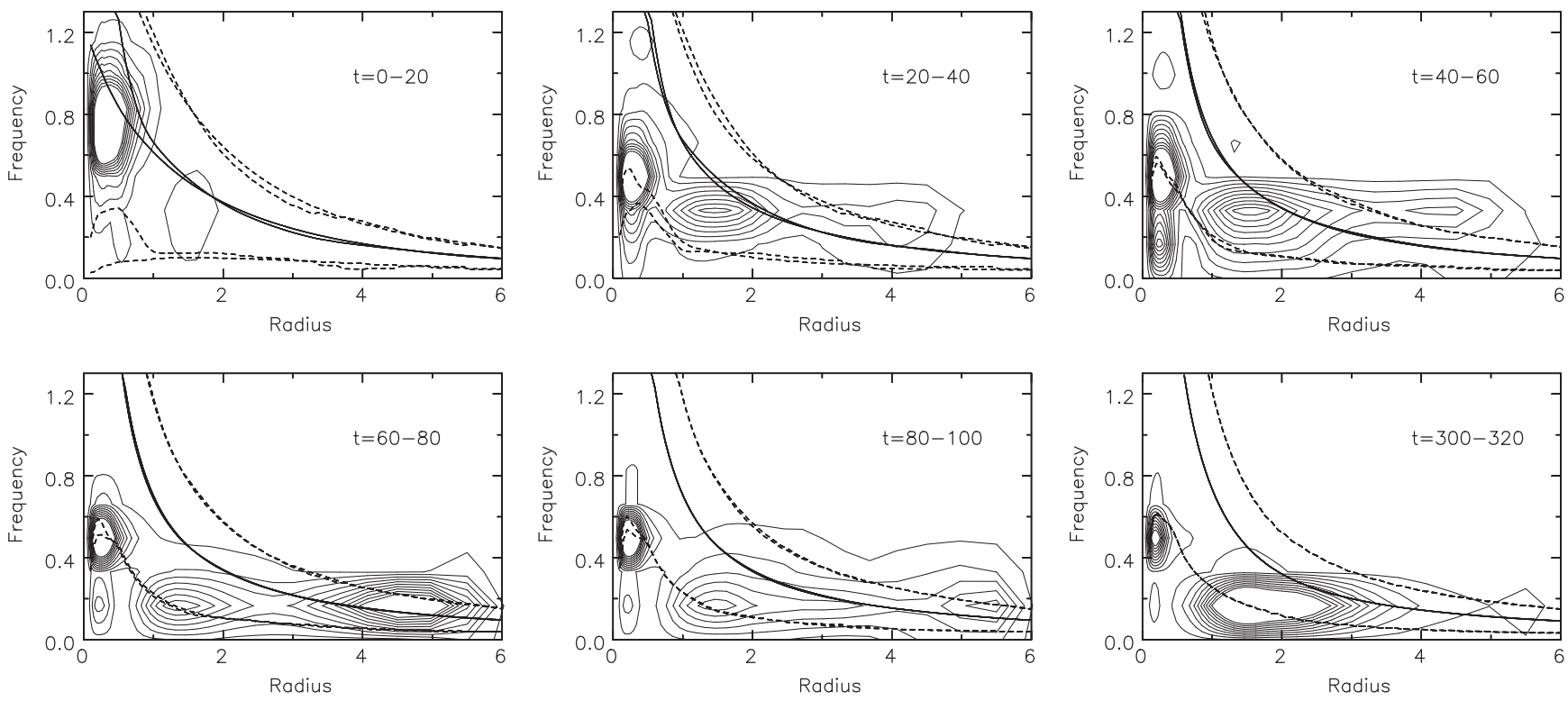

Figure 3. Power spectra of the $m=2$ Fourier component as a function of radius. The signals of the $m=2$ sectoral harmonic of the density are measured over a time interval of 20. The solid lines show $\Omega(R)$, the frequency of circular rotation. The dashed lines show $\Omega+\kappa / 2$ (upper) and $\Omega-\kappa / 2$ (lower), where $\kappa$ is the epicyclic frequency. The two sets of solid and dashed lines correspond to the curves of the starting and ending times in each interval, respectively. Generally, the lower sets denote the starting times, while the upper sets denote the ending times.

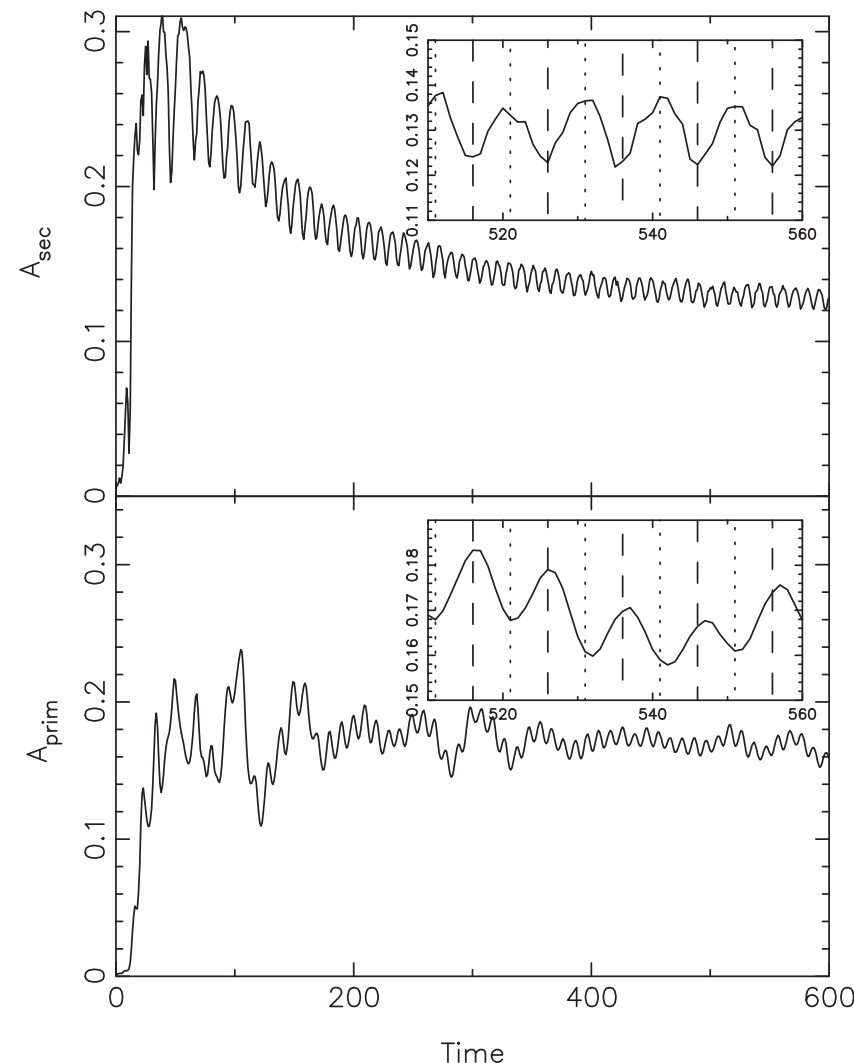

Figure 4. Time evolution of the amplitudes of the secondary bar (top panel) and the primary bar (bottom panel) of the standard S2B. $A_{\text {prim }}$ and $A_{\mathrm{sec}}$ are defined as the Fourier $m=2$ amplitude over the radial ranges $0.96 \leqslant R \leqslant 3.01$ and $R \leqslant 0.30$, respectively. In the insets, the dashed lines mark the times when the two bars are aligned, while the dotted lines mark the times when they are perpendicular.

bar amplitude is very small after $t=200$. This S2B structure persists to the end of the simulation, lasting for more than 6 Gyr. The two bars are not rigid bodies when they rotate

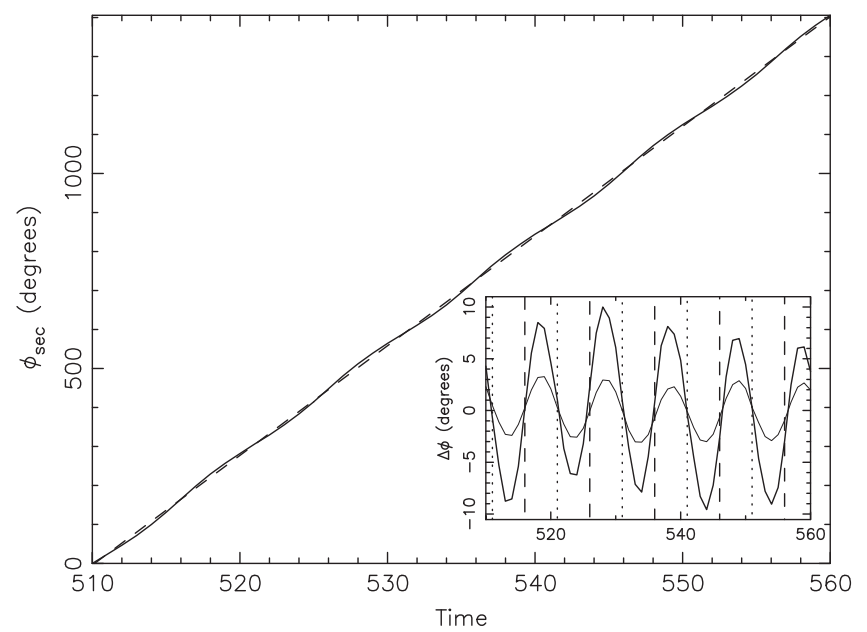

Figure 5. Time evolution of the phase of the secondary bar, measured between $t=510-560$. The dashed straight line is the least-square fit, which gives the slope $\left\langle\Omega_{\mathrm{sec}}\right\rangle$. The inset figure shows the phase difference, $\Delta \phi$, between the phases of the bars and $\langle\Omega\rangle$, where $\langle\Omega\rangle$ is the pattern speed averaged over one relative rotation of the two bars; the thick line with a large-amplitude oscillation is for the secondary bar while the thin line with a small-amplitude oscillation is for the primary bar. The dashed (dotted) lines mark the times that the two bars are parallel (perpendicular) to each other as in Figure 4.

through each other. As seen in the insets of Figure 4, the dotted and dashed lines match the local maximum or minimum points of bar amplitudes well, and they also match the inflection points of $\Delta \phi$ curves in the inset of Figure 5. The secondary bar is stronger and rotates slower when the two bars are perpendicular, while it is weaker but rotates faster when they are parallel (see also DS07). Generally, the secondary bar amplitude and pattern speed variations are larger than in the primary bar. Such oscillating pattern speeds and amplitudes are consistent with loop-orbit predictions (Maciejewski \& Sparke 2000).

In order to compare with observations, we use two methods to measure the bar length. As shown in the top panel of 

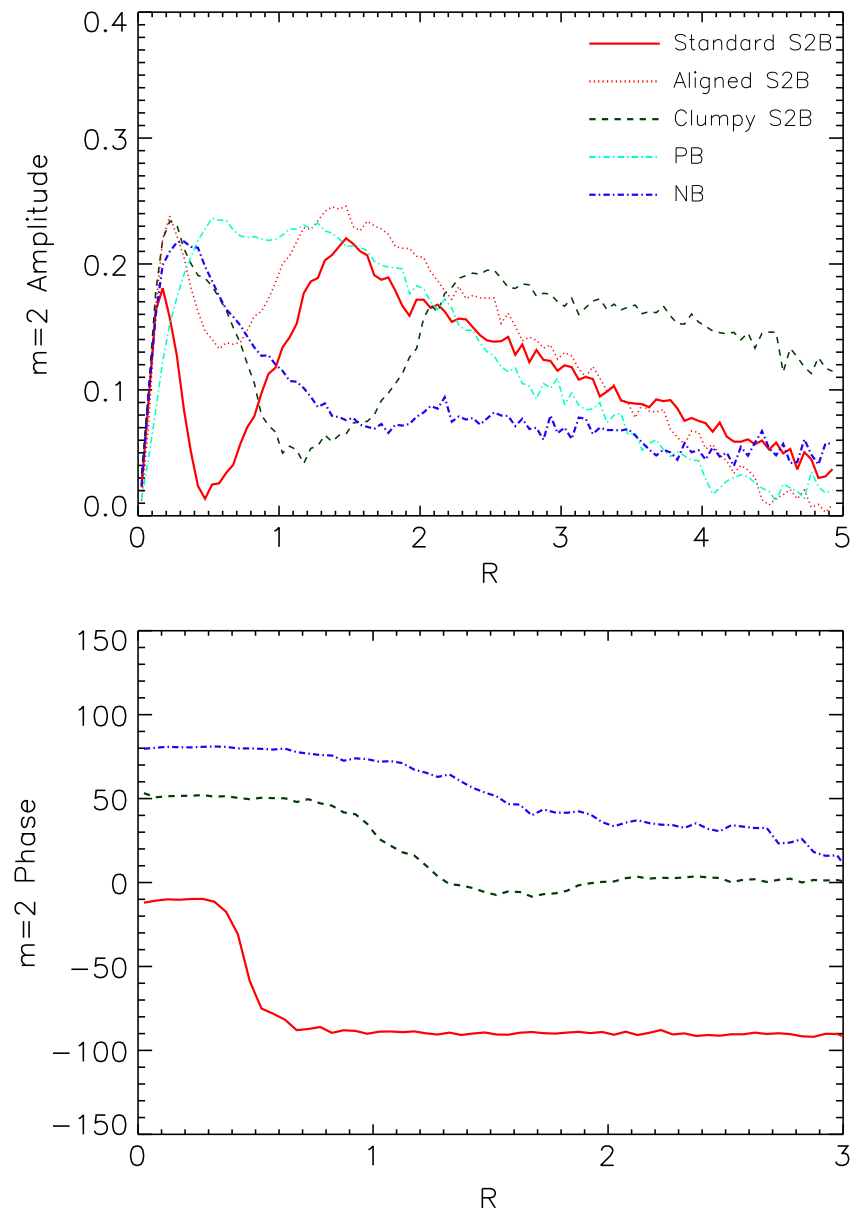

Figure 6. The $m=2$ amplitude and phase as a function of radius at $t=500$ when all structures are stable. Here we take the simulations discussed in this paper as representative of each type of models to show their bar strength and length. The phase of the small-scale bars in the standard S2B, clumpy S2B and NB models are shown in the bottom panel.

Figure 6, the secondary bar peak amplitude of the standard model is at $R \sim 0.2$. The length of the secondary bar, $a_{\mathrm{sec}} \sim 0.3$, is measured by tracing half-way down the peak on the $m=2$ amplitude plot, which is consistent with the value 0.4 given by the $10^{\circ}$ deviation from a constant phase. The semimajor axis of the primary bar, $a_{\text {prim }} \sim 3.0$, gives a length ratio $a_{\text {sec }} / a_{\text {prim }} \sim 0.10-0.13$ that is consistent with the typically observed length ratio of local S2B systems (median ratio 0.12; Erwin \& Sparke 2002; Erwin 2004; Lisker et al. 2006). The primary bar extends roughly to its co-rotation radius $\left(R_{\mathrm{CR} \text {,prim }} \sim 3.0 \sim a_{\text {prim }}\right)$, while the secondary bar is much shorter than its co-rotation radius $\left(R_{\mathrm{CR}, \mathrm{sec}} \sim 1.5\right)$. If $1.0 \leqslant R_{\mathrm{CR}} / a \lesssim 1.4$, the bar is classified as a "fast" bar (e.g., Debattista \& Sellwood 2000; Aguerri et al. 2003; Corsini et al. 2003; Debattista \& Williams 2004). Therefore, the primary bar is a "fast" bar, while the secondary bar is "slow." It is worth noting that the approximate co-rotation radius of the secondary bar $\left(R_{\mathrm{CR} \text {,sec }} \sim 1.5\right)$ is close to the outer ILR radius of the primary bar $\left(R_{\mathrm{oILR}, \text { prim }} \sim 1.5\right)$, which is considered as evidence of CR-ILR coupling by Rautiainen \& Salo (1999; see also Pfenniger \& Norman 1990; Friedli \& Martinet 1993).

Moreover, before the formation of the primary bar, the rapidly evolving inner disk exhibits recurrent transient spirals driven by the secondary bar as seen at $t=20$ in Figure 2. As the primary bar grows stronger, the nuclear spirals gradually disappear. Therefore, a reasonable inference is that the formation of the primary bar efficiently suppresses nuclear spirals.

\subsection{A Clumpy S2B Galaxy}

When $b_{Q} \lesssim 0.45$, all simulations experience a violent clumpy phase, shown by the shaded regions in Figure 1. As expected, with a colder inner disk, the clump instability becomes more significant. The clump instability occurs mainly at $R \lesssim 0.7$ where the initial $Q$ is less than 0.8 , which is roughly consistent with the criterion of Dekel et al. (2009; $Q \simeq 0.67$ ) for clump growth in an unstable disk. A thinner inner disk will also strengthen the clump instabilities, but thickness is not as important as $Q$. When $b_{Q} \gtrsim 0.7$, we never see clear clump instabilities even with a very thin inner disk. As shown in Figure 7, the inner disk fragments into clumps in a short time. Because of clump-clump gravitational interactions and dynamical friction, the massive clumps sink to the center and merge to form a small-scale bar. The polar grid code we adopt concentrates spatial resolution at the geometric center of the grid. However, the formation of clumps results in the highest density not coinciding with the region of highest spatial resolution. Therefore, we have tested refining the grid by doubling the number of radial and azimuthal grid cells; the result of this test is similar to the result shown here.

As with standard S2Bs, the two bars in clumpy S2Bs also form from independent bar instabilities of the inner and outer disks. Although the formation of the secondary bar is slightly delayed by the violent clumpy phase, as shown in Figure 8, the newly formed secondary bar can extend to its co-rotation radius as well, $R_{\mathrm{CR} \text {,sec }} \sim 1.0$. In our clumpy simulations, the clumpy phase always results in the formation of a small-scale bar at the beginning. However, they differ from standard S2Bs in terms of their dynamical properties. First, the amplitude evolution of the primary bar (Figure 9) shows that it forms much later and more gradually in the clumpy S2B. Second, the iso-density contours (Figure 7) show that the secondary bar is more disky and centrally concentrated, while the secondary bars of standard S2Bs tend to be more rectangular-shaped. We perform ellipse fitting for both models using IRAF, and find a more negative $B_{4}$ for the secondary bar of the standard S2B. In Figure 10, we show the central surface density profiles of the three models. Because they have the same disk mass $M_{d}=1.5$, they share the same density distribution at $t=0$. After the small-scale bar forms, at $t=80$, the central surface density of the clumpy S2B is $~ 35 \%$ larger than both the standard S2B and NB model. From $t=80$ to $t=500$, the surface density of all these three models increases at the same rate. Therefore, the extra central surface density of the clumpy S2B is produced during the clumpy phase. Third, the two bars of the clumpy S2B are both much longer than those of the standard $\mathrm{S} 2 \mathrm{~B}$ as shown in Figure 6. Measured by tracing half-way down the slope of $m=2$ amplitude, the length ratio is $a_{\text {sec }} / a_{\text {prim }} \sim 0.8 / 5.0=0.16$, slightly larger than standard $\mathrm{S} 2 \mathrm{Bs}$. If the bar length is measured at the radius where the $m=2$ phase deviates from a constant by $10^{\circ}, a_{\mathrm{sec}}$ is even larger at 1.0. Measured from Figure $8(t=200-220), R_{\mathrm{CR}, \mathrm{sec}}$ is about 1.3 , then $R_{\mathrm{CR}, \mathrm{sec}} / a_{\mathrm{sec}} \sim 1.3-1.6$. Thus the secondary bar is a quite "fast" bar extending close to its co-rotation radius, which is quite common in our clumpy S2Bs. This surprising result demonstrates that it is dynamically possible to generate a 

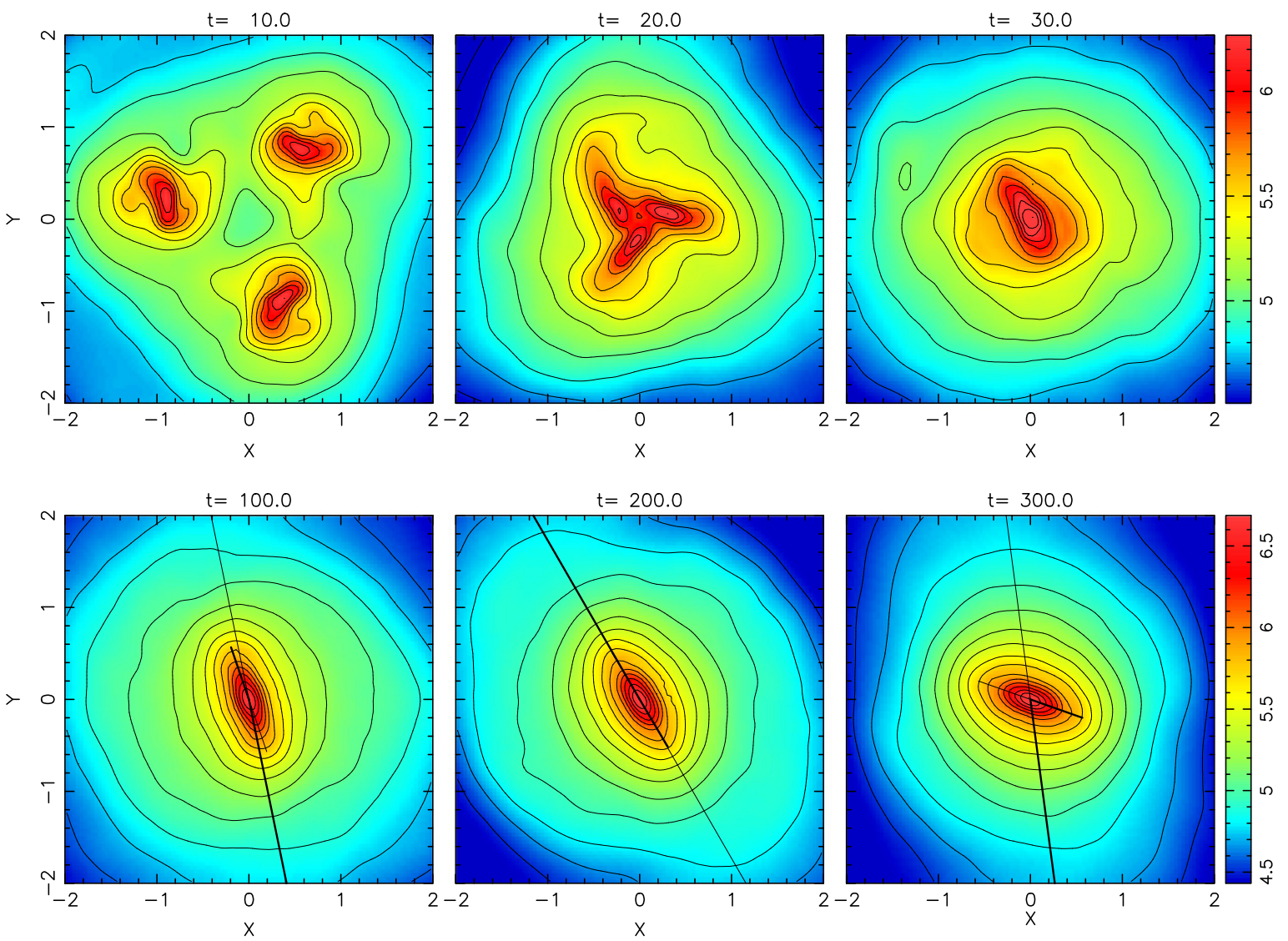

Figure 7. Formation process of a double-barred galaxy from a clumpy phase. This simulation has $M_{d}=1.5$ and $b_{Q}=0.3$.
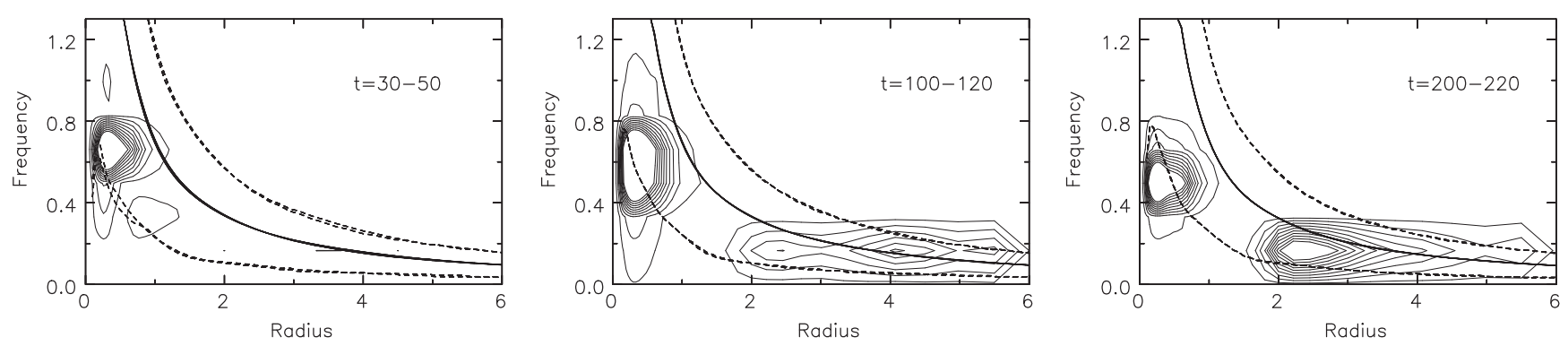

Figure 8. Power spectra of the $m=2$ Fourier component as a function of radius for the clumpy model in Figure 7 .

stable "fast" S2B system. As shown in the top panel of Figure 9, the change of $A_{\mathrm{sec}}$ is quite small with the formation of the primary bar. Moreover, the two bars oscillate in the same way as standard S2Bs, but the oscillation is smaller. These indicate the interaction of two bars is weaker. In Figure 7 $(t=200,300)$, we can clearly see that the inner disk is dominated by the secondary bar. Thus the primary bar is not efficient at trapping the secondary bar in the clumpy S2B models. A possible reason is that the torques from the primary bar are not strong enough to reduce the strength and length of the secondary bar, because the primary bar is much fatter and less massive than the ones of standard S2Bs. This is because the primary bar forms further out in the disk. Moreover, a more massive and concentrated secondary bar may also stabilize it against the primary bar. As shown in Figure 10, in the very central region the clumpy $\mathrm{S} 2 \mathrm{~B}$ is more massive than the standard S2B. In conclusion, in our clumpy S2Bs, the primary bar is too weak to be efficient at trapping the secondary bar, in which case a "fast" S2B system can be sustained for a long time.

These clumpy S2B simulations demonstrate that a smallscale bar can be generated from a violent clumpy phase. Previous $N$-body+gas simulations showed that clumps coalesce into a bulge whose shape was bar-like (Elmegreen et al. 2008, Figures 2 and 3). Inoue \& Saitoh (2012) showed that clumpyorigin bulges are bar-like in their hydrodynamic (SPH) simulations, and have exponential surface density profile, boxy (bar-like) shape and significant rotation. All these properties are consistent with the small-scale bars in our simulations. Therefore, these "bar-like" structures may be similar to our small-scale bars, which suggests that the secondary bar might often form from clump mergers.

\subsection{An Aligned S2B Galaxy}

Figures 11 and 12 show the formation and coupling process of an aligned S2B. As shown in Figure 12, the initial bar 


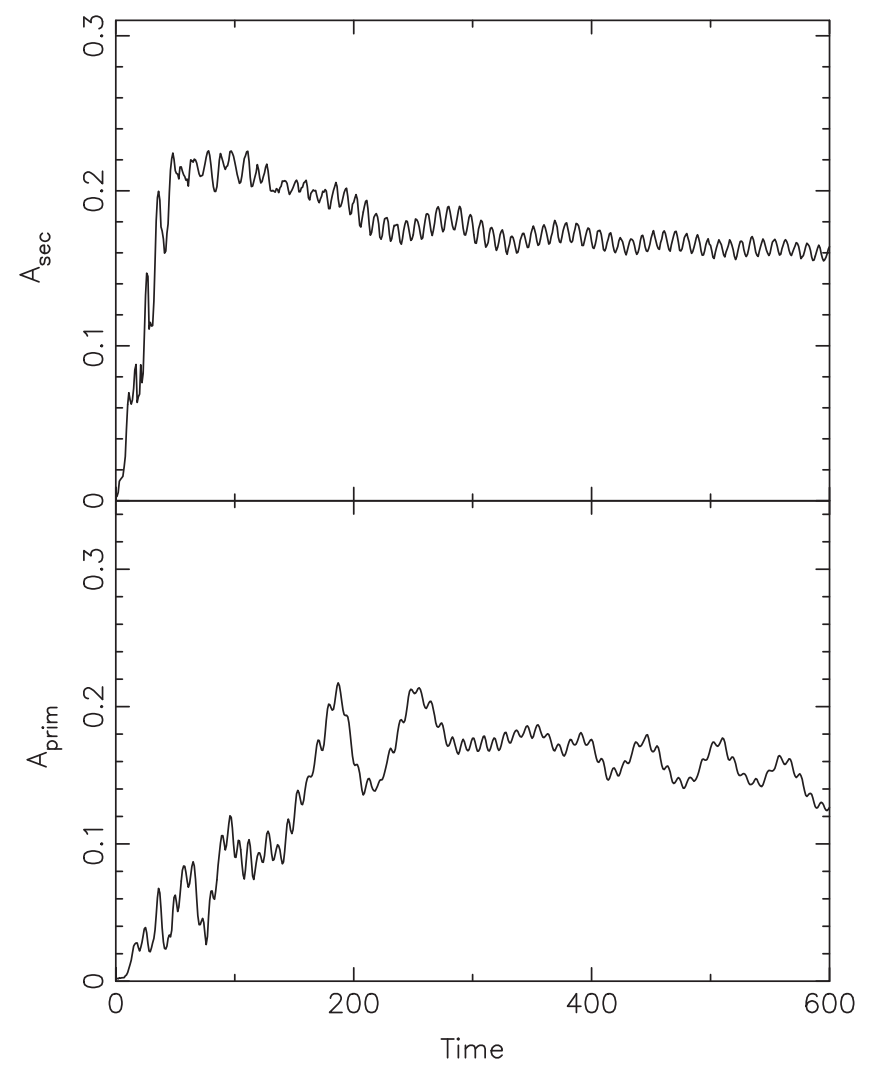

Figure 9. Time evolution of the amplitude of the secondary bar (top panel) and the primary bar (bottom panel) of the clumpy S2B. $A_{\text {prim }}$ and $A_{\text {sec }}$ are defined as the Fourier $m=2$ amplitude over the radial ranges $2.00 \leqslant R \leqslant 4.07$ and $R \leqslant 0.96$, respectively.

instabilities form two bars with different pattern speeds between $t=0$ and 80 . The oscillating properties are identical to those of the standard and clumpy S2Bs. During the period $t=80-270$, the secondary bar gradually becomes shorter and weaker because of the interaction with the primary bar. At $t \simeq 280$ (Figure 11) the secondary bar cannot pass through the next perpendicular barrier where the rotational kinetic energy of the secondary bar is minimal due to the gravitation torque from the primary bar. Then the pattern speed of the secondary bar decreases sharply to that of the primary bar. The secondary bar remains at a fixed orientation relative to the primary bar during $t=280-300$. The two bars share the same pattern speed, although they are still mis-aligned at a roughly constant angle. During this time, the surface density contours around the secondary bar become somewhat peanut-shaped. This shape transformation always happens in all our aligned S2Bs. Then the secondary bar falls back to alignment with the primary bar gradually, which is the lowest energy state. Sometimes, the secondary bar librates slightly around the major axis of the primary bar before complete alignment. Finally, they rotate at the same pattern speed until the end of the simulation. Generally, this coupling period occurs over a few tens of time units ( 100 Myr).

Although the aligned S2B now resembles a single-barred galaxy, the relic of the secondary bar can be clearly seen as a nuclear peak in the $m=2$ amplitude profile (Figure 6). The peanut-shaped contours are another signature of aligned S2Bs that survive to the end of our simulations. Normally, the coupling process is dominated by the primary bar as the secondary bar gradually becomes shorter and weaker. Only

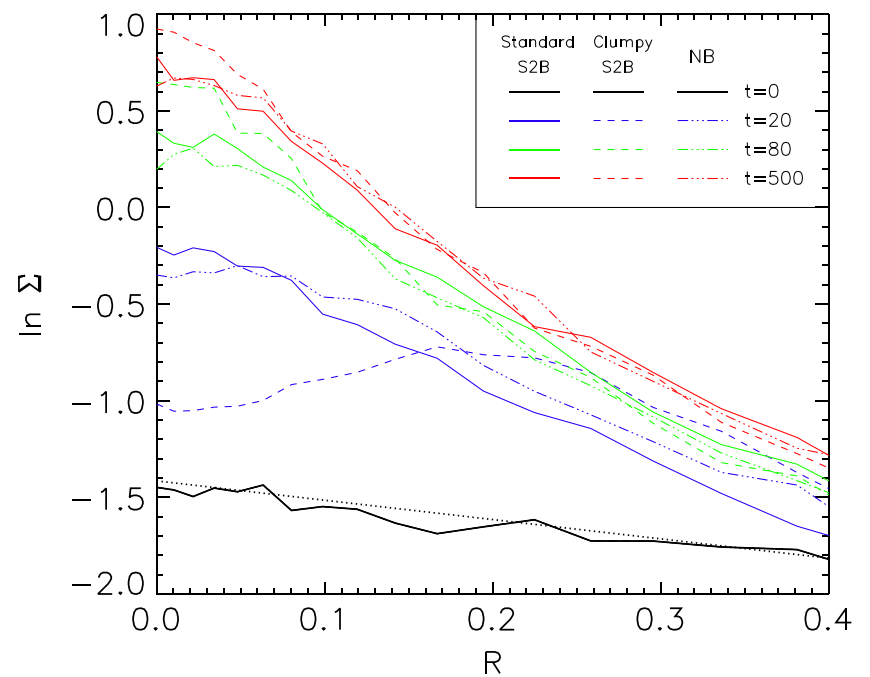

Figure 10. Central surface density profiles of the standard S2B, clumpy S2B and NB models at $M_{d}=1.5$ highlighted by the dashed black rectangle on Figure 1. The black line is their initial surface density profile.

very few models show a coupling process dominated by the secondary bar, in which the primary bar is nearly destroyed by the secondary bar during their coupling process. A fraction of the particles previously belonging to the primary bar are, in this case, trapped around the secondary bar, making it longer.

For our standard S2Bs, the pattern speed ratio of the two bars, $\Omega_{\text {prim }} / \Omega_{\text {sec }}$, varies from 0.36 to 0.55 , while it is $0.25-0.43$ for the clumpy S2Bs. Generally, the pattern speeds of the primary bars of the clumpy S2Bs are lower than of the standard S2Bs. Before alignment, the pattern speed of the aligned S2Bs is $0.35-0.49$, i.e., similar to the range of the standard S2Bs. Therefore, it is hard to predict whether a S2B system will couple or not based only on its pattern speed ratio. Possibly, the mass and morphology of two bars also play a role.

\section{LARGE-SCALE PB GALAXIES AND NB GALAXIES}

When $b_{Q} \geqslant 0.8$, most of the simulations form large-scale single-barred galaxies, PBs, as can be seen in Figure 1. Compared with the formation process of S2Bs, the bar instability starts with a lower pattern speed. By losing angular momentum to the outer disk, the bar gradually slows down. Then its co-rotation radius is pushed further out and more particles at larger radius can be trapped forming a longer bar (Athanassoula 2003). In this way, they cannot form two independently rotating bars.

A fraction of simulations only leave small-scale bars after their weak primary bars are gradually dissolved, or, in some cases, never formed at all. These models are marked as "NB" in Figure 1. Although NBs are also a type of single-barred galaxy, compared with the PBs, they have different formation histories and properties. To form NBs, a mechanism is needed to prevent the mass of the outer disk from being trapped into the primary bar. The initial $Q$ of the outer disk is about 2.0, which is close to the criterion (2.0-2.5) for non-axisymmetric stability in all disk mass distributions (Athanassoula \& Sellwood 1986). To quantify how sensitive the formation of the primary bar is to the change of $Q$, we fix the $Q$ profile of the inner disk, and vary $Q$ in the outer disk from 2.0 to 2.3 . If $Q \geqslant 2.2$, the outer disk is unresponsive to bar instabilities, showing that bar formation is very sensitive to $Q$ when $Q$ is large. Even a small enhancement 

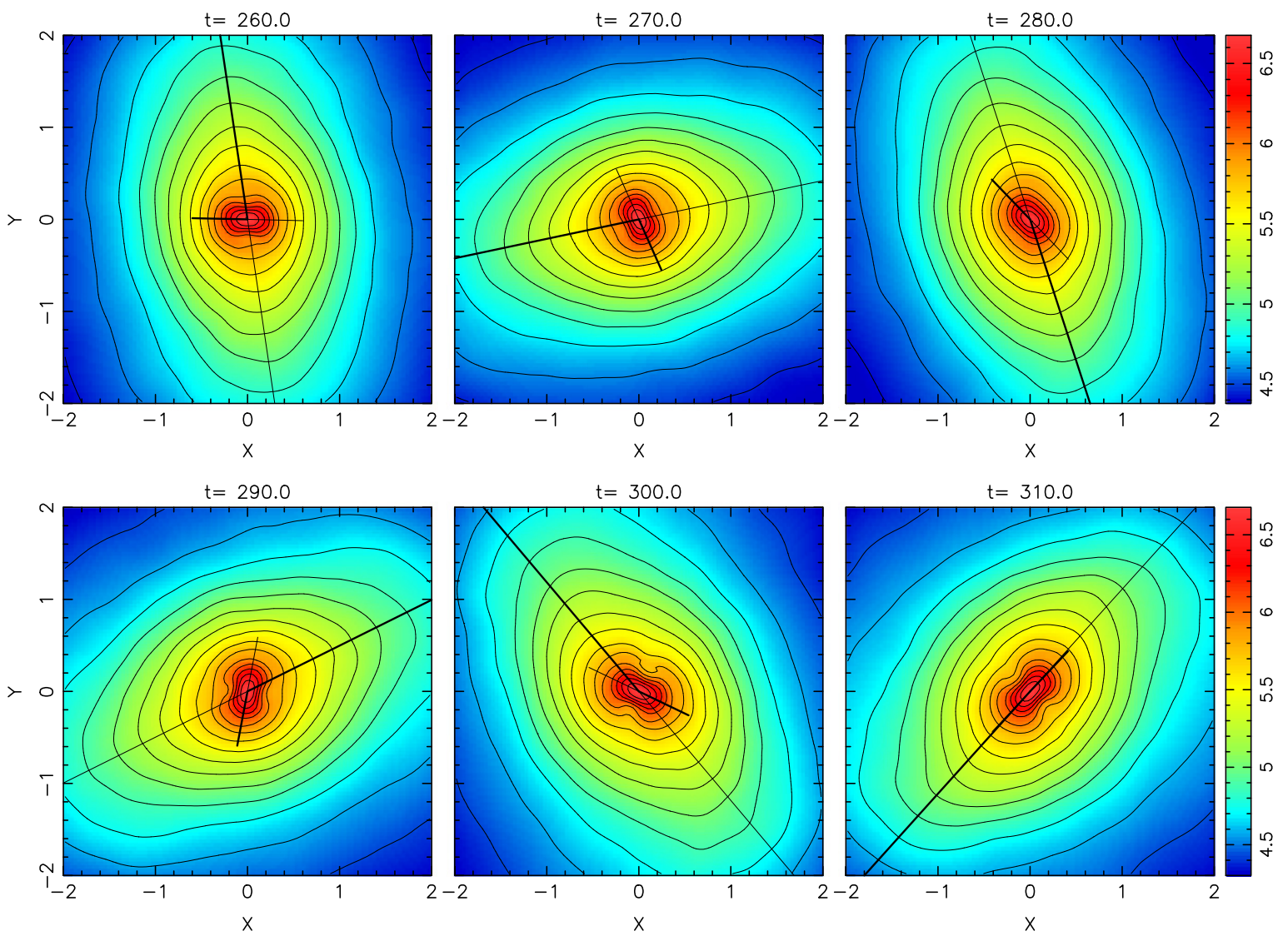

Figure 11. Coupling phase of the two bars in the aligned S2B case. This simulation has $b_{Q}=0.5$ and $M_{d}=1.1$. Note the developing of peanut-shaped contours from $t=290$.

(An animation of this figure is available.)
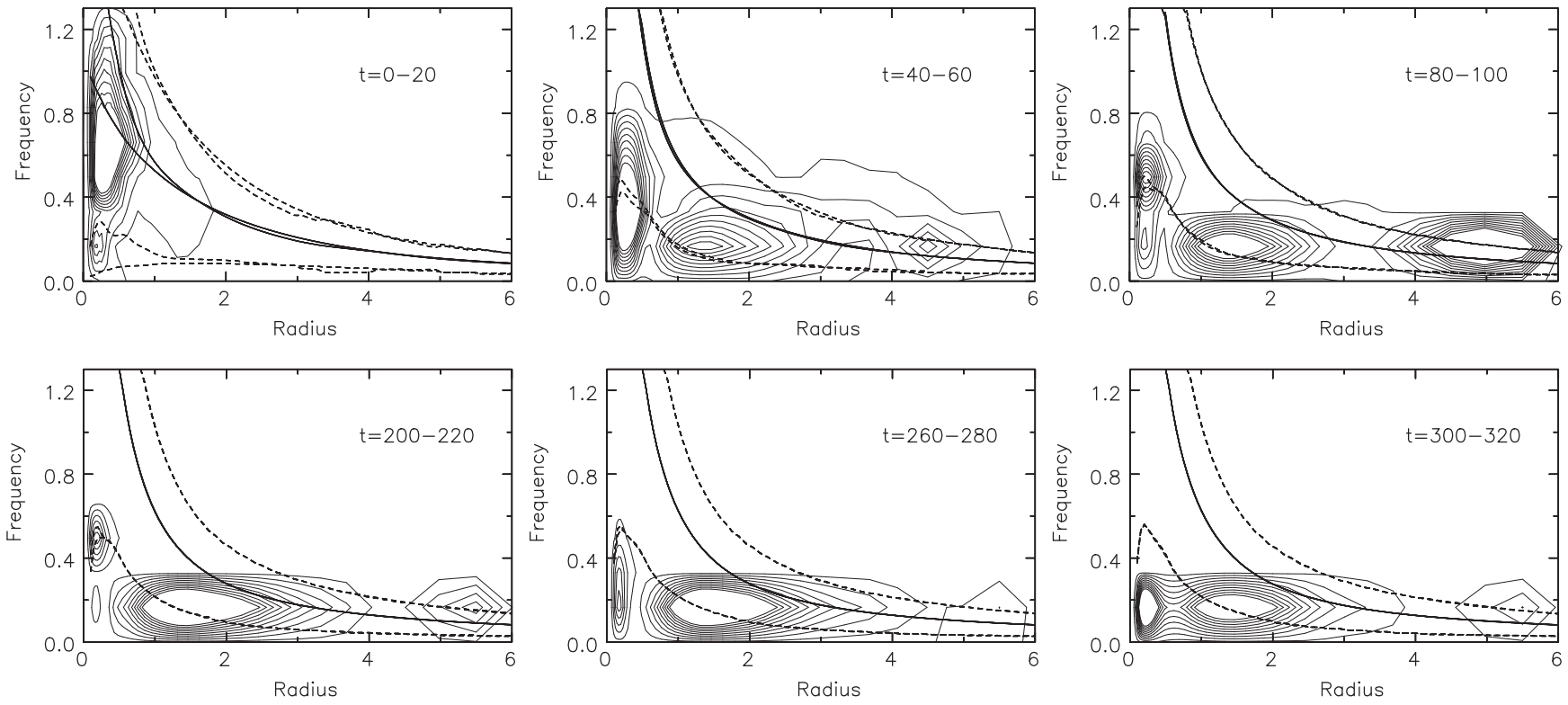

Figure 12. Power spectra of the $m=2$ Fourier component as a function of radius for the aligned S2B model in Figure 11.

of $Q$ in the hot outer disk can significantly suppress the formation of the primary bar.

For the subsamples of NBs, standard S2Bs, and clumpy S2Bs, we show the evolution of their azimuthally averaged $Q$, $\langle Q\rangle$, in Figure 13. From $t=100$ to $t=500$, the changes in the inner disks are very small while the outer disks of the NBs become hotter than both standard and clumpy S2Bs. Such dynamically hot outer disks become unsuitable to form a steady primary bar. In some cases, a weak and sometimes spiral-like primary bar forms in the early phase, but it is hard to stabilize 


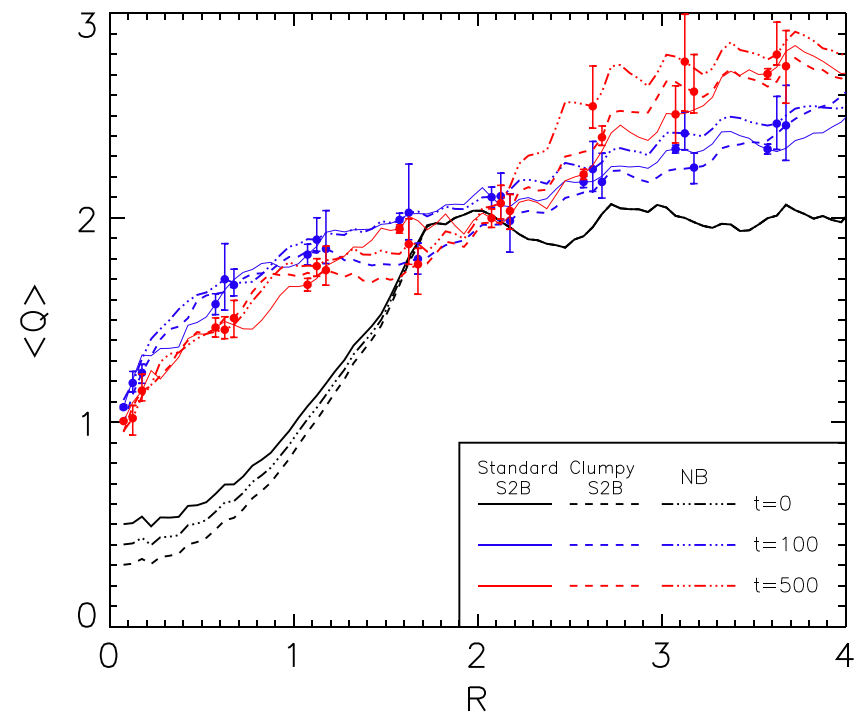

Figure 13. Evolution of the mean value of $Q$ of standard S2Bs, clumpy S2Bs, and NBs that correspond to the three groups of models in Figure 1 within the red, blue, and green rectangles, respectively. The black lines are the initial $Q$ profiles of these simulations. The error bars refer to the scatter of each group of simulations.

for a long time. One possible explanation for the enhancement of $Q$ is the spirals driven by the nuclear bar. A lot of studies have shown that spirals efficiently heat disks to more eccentric orbits (Barbanis \& Woltjer 1967; Bertin \& Lin 1996; Carlberg \& Sellwood 1985; Binney \& Lacey 1988; Jenkins \& Binney 1990; De Simone et al. 2004; Minchev \& Quillen 2006). In our simulations, we do see stronger spirals recur more frequently in NBs than in standard and clumpy S2Bs.

\section{DISCUSSION AND CONCLUSIONS}

We have been successful in forming S2B galaxies with a set of simple initial conditions involving a cooler inner disk. By setting up an increasingly cooler disk toward the center, an S2B structure can form naturally. Recent $N$-body+gas simulations by Wozniak (2015) showed that the star formation in the central regions was responsible for stabilizing the nuclear bars. New stars formed a dynamically cool inner disk where nuclear bar instabilities arose, consistent with our results. Rautiainen \& Salo (1999) and Rautiainen et al. (2002) found that the secondary bar formed from the highly rotating central mode, and models with steeply rising rotation curves tended to have a long-lasting secondary bar. DS07 used a pre-existing rapidly rotating pseudo-bulge to generate $\mathrm{S} 2 \mathrm{Bs}$. All these simulations suggest that a rapidly rotating nuclear component might plausibly generate a small-scale bar with high pattern speed. The two bars form from independent bar instabilities of the inner and outer parts of disks. Thus the pattern speeds of the two bars differ significantly in such S2Bs. Saha \& Maciejewski (2013) generated an S2B in a galaxy model whose gravitational potential was dominated by the dark matter halo. The two bars rotated at comparable pattern speed, but they developed from two independent bar instabilities as well. Therefore, we conclude that $\mathrm{S} 2 \mathrm{Bs}$ can form by simply generating independent bar instabilities in the inner and outer disks, although the survival of S2B systems is stochastic because of the chaotic nature of disks. A dynamically cool inner disk embedded in a hotter outer disk is an easy and natural way to satisfy this condition.

In the early phase of the formation of S2Bs, the secondary bars extend to their co-rotation radius, which indicates that they form from the usual bar instabilities as with the primary bars. The angular momentum exchange among the hosting disk, secondary bar, and primary bar, determines the strength, morphology, and final pattern speeds of the two bars. The primary bars extend roughly to their co-rotation radius, so they are "fast" bars. For the standard S2Bs, although the secondary bars extend to the co-rotation radius at formation, they shrink to a much smaller size to become "slow" bars because of the suppression from the later-formed primary bars. The clumpy $\mathrm{S} 2 \mathrm{Bs}$ show that the secondary bar can form from the violent clumpy phase. With a weaker primary bar, the clumpy S2B can host a "fast" secondary bar. Because of the interaction of the two bars and the disk, there are also a large fraction of unstable $\mathrm{S} 2 \mathrm{Bs}$, with the two bars coupling to alignment in just a few Gyr.

In our models, the secondary bars always form earlier than the primary ones, which is consistent with previous collisionless simulations (e.g., Rautiainen et al. 2002; DS07). However, because the whole galaxy is set up at the beginning, we cannot constrain the cold material accumulation of the cool inner disk. It is not entirely clear to us how a dynamically cool inner disk, required in forming S2Bs, may arise. It is generally thought that gas is driven from outside in (e.g., a possible channel is provided by the primary bar). In this case, gas accumulated near the center can give rise to a cool disk that may be able to form a nuclear bar. However, an alternative formation scenario is that the secondary bar forms from the violent clumpy phase in the early universe when the primary bar is still not formed. In many $\mathrm{N}$-body $+\mathrm{SPH}$ simulations, these clumpy-origin smallscale bars could be easily mistaken for bulges. Therefore, the question remains whether the small-scale bar instabilities happens at the early time of the galaxy formation or after the formation of the primary bar. To better understand the formation of S2Bs, further numerical simulations are required.

We thank Bruce Elmegreen and Witold Maciejewski for insightful comments and discussions on the manuscript. Min Du thanks the Jeremiah Horrocks Institute of the University of Central Lancashire for their hospitality during a three-month visit while this paper was in progress. Hospitality at APCTP during the 7th Korean Astrophysics Workshop is kindly acknowledged. The research presented here is partially supported by the 973 Program of China under grant No. 2014CB845700, by the National Natural Science Foundation of China under grant Nos.11333003, 11322326, and 11073037, and by the Strategic Priority Research Program "The Emergence of Cosmological Structures" (No. XDB09000000) of Chinese Academy of Sciences. This work made use of the facilities of the Center for High Performance Computing at Shanghai Astronomical Observatory. V.P.D. is supported by STFC Consolidated grant No. ST/J001341/1.

\section{REFERENCES}

Aguerri, J. A. L., Debattista, V. P., \& Corsini, E. M. 2003, MNRAS, 338, 465 Athanassoula, E. 2003, MNRAS, 341, 1179

Athanassoula, E., \& Sellwood, J. A. 1986, MNRAS, 221, 213

Barbanis, B., \& Woltjer, L. 1967, ApJ, 150, 461

Bertin, G., \& Lin, C. C. 1996, Spiral Structure in Galaxies a Density Wave Theory, Vol. 271 (Cambridge, MA: MIT Press) 
Binney, J., \& Lacey, C. 1988, MNRAS, 230, 597

Buta, R., \& Crocker, D. A. 1993, AJ, 105, 1344

Carlberg, R. G., \& Sellwood, J. A. 1985, ApJ, 292, 79

Combes, F. 1994, in Mass-Transfer Induced Activity in Galaxies, ed. I. Shlosman (Cambridge: Cambridge Univ. Press), 170

Corsini, E. M., Debattista, V. P., \& Aguerri, J. A. L. 2003, ApJL, 599, L29

Dekel, A., Sari, R., \& Ceverino, D. 2009, ApJ, 703, 785

de Lorenzo-Cáceres, A., Falcón-Barroso, J., \& Vazdekis, A. 2013, MNRAS, 431, 2397

de Lorenzo-Cáceres, A., Falcón-Barroso, J., Vazdekis, A., \& Martínez-Valpuesta, I. 2008, ApJL, 684, L83

Debattista, V. P., \& Sellwood, J. A. 2000, ApJ, 543, 704

Debattista, V. P., \& Shen, J. 2007, ApJL, 654, L127

Debattista, V. P., \& Williams, T. B. 2004, ApJ, 605, 714

De Simone, R., Wu, X., \& Tremaine, S. 2004, MNRAS, 350, 627

de Vaucouleurs, G. 1975, ApJS, 29, 193

Elmegreen, B. G., Bournaud, F., \& Elmegreen, D. M. 2008, ApJ, 688, 67

Englmaier, P., \& Shlosman, I. 2004, ApJL, 617, L115

Erwin, P. 2004, A\&A, 415, 941

Erwin, P. 2005, MNRAS, 364, 283

Erwin, P., \& Sparke, L. S. 2002, AJ, 124, 65

Friedli, D., \& Martinet, L. 1993, A\&A, 277, 27

Heller, C., Shlosman, I., \& Englmaier, P. 2001, ApJ, 553, 661

Heller, C. H., Shlosman, I., \& Athanassoula, E. 2007, ApJL, 657, L65

Inoue, S., \& Saitoh, T. R. 2012, MNRAS, 422, 1902

Laine, S., Shlosman, I., Knapen, J. H., \& Peletier, R. F. 2002, ApJ, 567, 97

Lisker, T., Debattista, V. P., Ferreras, I., \& Erwin, P. 2006, MNRAS, 370, 477
Louis, P. D., \& Gerhard, O. E. 1988, MNRAS, 233, 337

Lynden-Bell, D., \& Kalnajs, A. J. 1972, MNRAS, 157, 1

Jenkins, A., \& Binney, J. 1990, MNRAS, 245, 305

Maciejewski, W., \& Athanassoula, E. 2007, MNRAS, 380, 999

Maciejewski, W., \& Sparke, L. S. 1997, ApJL, 484, L117

Maciejewski, W., \& Sparke, L. S. 2000, MNRAS, 313, 745

Miller, R. H. 1964, ApJ, 140, 250

Minchev, I., \& Quillen, A. C. 2006, MNRAS, 368, 623

Petitpas, G. R., \& Wilson, C. D. 2004, ApJ, 603, 495

Pfenniger, D., \& Norman, C. 1990, ApJ, 363, 391

Rautiainen, P., \& Salo, H. 1999, A\&A, 348, 737

Rautiainen, P., Salo, H., \& Laurikainen, E. 2002, MNRAS, 337, 1233

Saha, K., \& Maciejewski, W. 2013, MNRAS, 433, L44

Shen, J., \& Debattista, V. P. 2009, ApJ, 690, 758

Sellwood, J. A. 1989, MNRAS, 238, 115

Sellwood, J. A. 2014, arXiv:1406.6606

Sellwood, J. A., \& Debattista, V. P. 2009, MNRAS, 398, 1279

Sellwood, J. A., \& Valluri, M. 1997, MNRAS, 287, 124

Shlosman, I., Begelman, M. C., \& Frank, J. 1990, Natur, 345, 679

Shlosman, I., Frank, J., \& Begelman, M. C. 1989, Natur, 338, 45

Shlosman, I., \& Heller, C. H. 2002, ApJ, 565, 921

Silverman, B. W. 1986, Monographs on Statistics and Applied Probability (London: Chapman and Hall)

Toomre, A. 1981, in The Structure and Evolution of Normal Galaxies, ed. S. M. Fall \& D. Lynden-Bell (Cambridge: Cambridge Univ. Press), 111 Wozniak, H. 2015, A\&A, 575, A7 